\title{
Growth of Large-Area Aligned Molybdenum Nanowires by High Temperature Chemical Vapor Deposition: Synthesis, Growth Mechanism, and Device Application
}

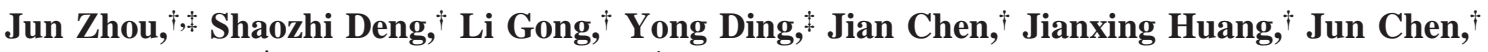 \\ Ningsheng Xu, ${ }^{*}$, and Zhong Lin Wang*,* \\ State Key Lab of Optoelectronic Materials and Technologies, and Guangdong Province Key Laboratory of \\ Display Materials and Technologies, School of Physics and Engineering, SunYat-Sen (Zhongshan) University, \\ Guangzhou, 510275, China, and School of Materials Science and Engineering, Georgia Institute of Technology, \\ Atlanta, Georgia 30332-0245
}

Received: February 25, 2006; In Final Form: April 6, 2006

\begin{abstract}
Large-area aligned Mo nanowires have been grown on stainless steel substrates by high-temperature chemical vapor deposition with the use of Mo metal. The detailed physical and chemical growth processes regarding the formation of the nanowires have been investigated using mass spectroscopy, thermogravimetry, and differential scanning calorimetry analysis, as well as structure analysis by electron microscopy. In reference to Gibbs energy calculation, our study reveals that the growth relies on the decomposition of $\mathrm{MoO}_{2} \mathrm{vapors}$ through condensation of its vapor at high substrate temperatures. The aligned growth is a result of competing growth with the nanowires normal to the substrate surface reaching the final growth front. The field emission measurement and the vacuum luminescent tube study show that the Mo nanowires have potential application as electron emitters.
\end{abstract}

\section{Introduction}

Investigation of metal nanowires has attracted much attention due to their wide range of potential applications in areas such as nanoscale circuitry linkages, field electron emitters, nanosensors, magnetic devices, and many more. ${ }^{1}$ Various methods have been developed for preparation of metal nanowires, including template assisted electrochemical deposition, ${ }^{2}$ chemical vapor deposition, ${ }^{3}$ replacement reactions, ${ }^{4}$ metal compound reduction, ${ }^{5,6}$ metal organic decomposition, ${ }^{7}$ physical/chemical vapor deposition, ${ }^{8}$ and sputter deposition. ${ }^{9}$ Many important applications require direct growth of aligned metal nanowire arrays on a large-area substrate. The template assisted method has been proven successful for preparing aligned metal nanowires..$^{2 j}$

Molybdenum (Mo) has been an important cathode material for field emission arrays (FEAs), due to its good thermal, mechanical, and electrical properties. ${ }^{10}$ Mo nanowires have been prepared by reducing molybdenum oxide nanowires with $\mathrm{H}_{2}$ gas. ${ }^{6 \mathrm{~d}-\mathrm{f}}$ However, the reduction process degrades the crystallinity of the nanowires and the adhesion between nanowires and substrate, limiting its application in devices. For technological applications, it is highly desirable to grow aligned Mo nanowires in a single-step on large-area and conductive substrates. Recently, Vaddiraju et al. ${ }^{8 \mathrm{c}}$ reported that $\mathrm{W}$ nanowires can be directly synthesized by heating tungsten (W) filaments to high temperature where the tungsten oxides can decompose. In this paper, we scaled and improved the method and synthesized large-quantity aligned Mo nanowire in flat-surfaces

* Corresponding authors. E-mail: stsxns@zsu.edu.cn (N.X.); zhong.wang@mse.gatech.edu (Z.L.W.).

† State Key Lab of Optoelectronic Materials and Technologies, and Guangdong Province Key Laboratory of Display Materials and Technologies, School of Physics and Engineering, SunYat-Sen (Zhongshan) University.

$\doteqdot$ School of Materials Science and Engineering, Georgia Institute of Technology. of stainless steel and silicon substrates. The chemical process involved in the growth has been systematically investigated in a conjunction use of several analytical techniques. A growth mechanism has been proposed based on the observed microstructures. Finally, the field emission properties of the Mo nanowires and their application in cold cathode vacuum luminescent tubes have been demonstrated.

\section{Experimental Section}

Synthesis Apparatus and Nanowire Growth. The synthesis apparatus (see Supporting Information) uses a bell jar vacuum chamber $(\phi 300 \mathrm{~mm} \times 400 \mathrm{~mm})$ evacuated by using a rotary pump, in the center of which a Mo boat as evaporation source was heated by electric current. There is also a gas inlet control system and an infrared pyrometer for temperature monitoring. Stainless steel foils ( $\Phi 1.6 \mathrm{~mm}$, disk form), Si strips or singlecrystalline $\mathrm{Al}_{2} \mathrm{O}_{3}$ strips were used as the substrates. Alumina strips were first placed in the Mo boat, and then the substrates were placed on the top surface of the alumina strips. The metal nanowires will grow on the top surface of the substrate. After evacuation of the chamber to less than $5 \mathrm{~Pa}$, argon (Ar) gas (99\%) was introduced into the synthesis system at a flow rate of $200 \mathrm{sccm}$ (standard cubic centimeters per minute). Twenty minutes later, the peak temperature of the boat was elevated to $\sim 1623 \mathrm{~K}$ or even higher at a rate of $100 \mathrm{~K} / \mathrm{min}$, and the temperature of the substrates was at $\sim 1453 \mathrm{~K}$ or higher. The boat and the substrates were maintained at the designed temperature for 20 min under a pressure of $\sim 80 \mathrm{~Pa}$ before the boat was allowed to cool to room-temperature quickly, i.e., from $\sim 1623$ to $\sim 673 \mathrm{~K}$ within $3 \mathrm{~min}$. Then, hydrogen gas was introduced to the synthesis system at a flow rate of $100 \mathrm{sccm}$ during the cooling process. However, we noticed that no nanowires were grown if hydrogen was flowing throughout the entire synthesis process. 


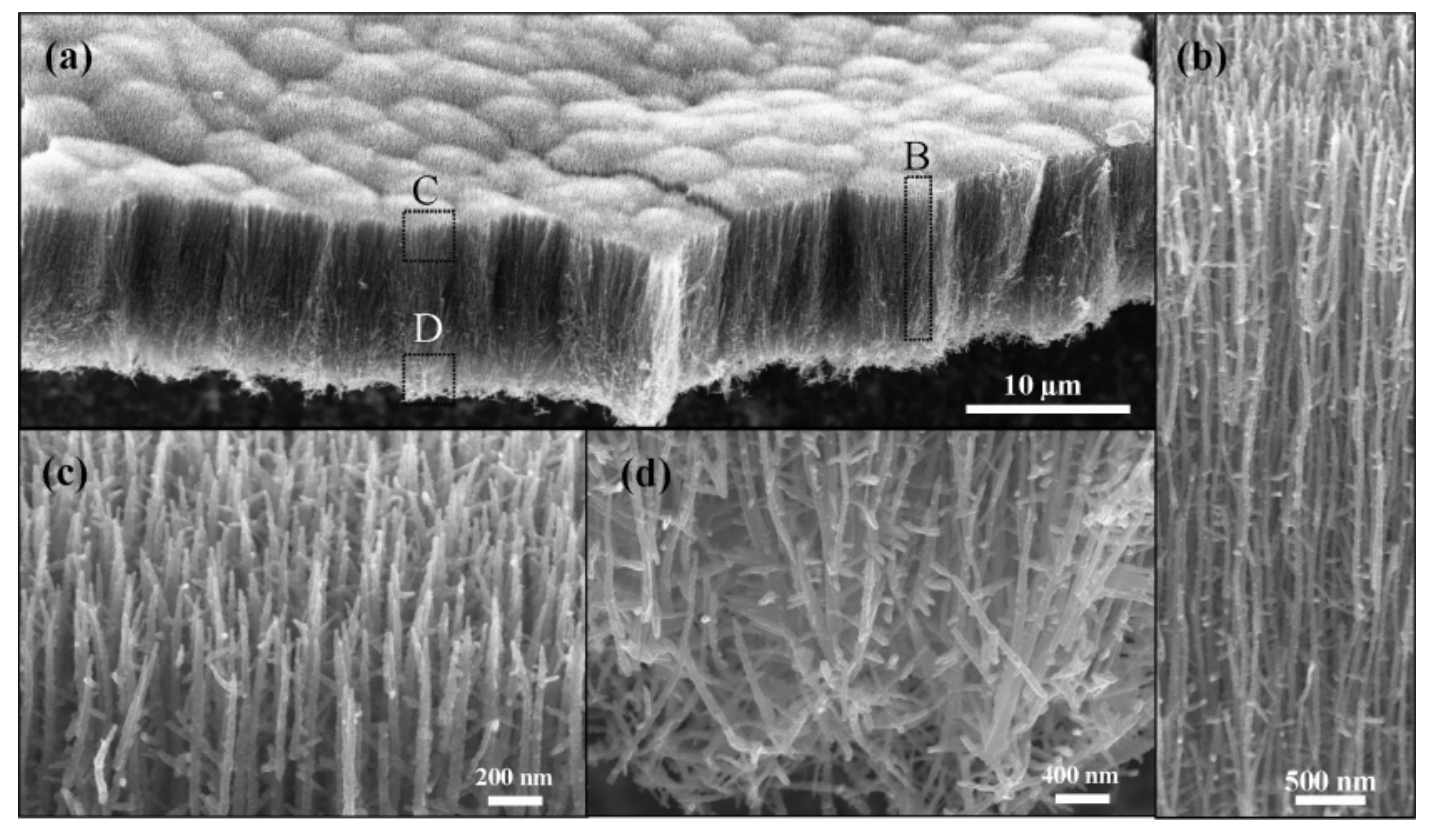

Figure 1. (a) Low magnification SEM image of Mo nanowires. (b-d) high magnification SEM images of rectangular-enclosed areas B-D in part a, respectively.

Structure Analysis. The synthesized products were characterized by X-ray diffraction spectroscope (XRD) (D/max-3A apparatus with $\mathrm{Cu} \mathrm{K} \alpha$ radiation), high-resolution field emission scanning electron microscopy (SEM) (Leo 1530 FEG at 5 and $10 \mathrm{kV}$ ), transition electron microscopy (TEM) (JEOL 100C at $100 \mathrm{kV}$ and Hitachi HF2000 at $200 \mathrm{kV}$ ), high-resolution transition electron microscopy (HRTEM) (JEOL 4000EX at 400 $\mathrm{kV}$ ), and energy-dispersive X-ray spectroscope (EDS) attached to the SEM.

TG and DSC Characterization. To obtain the information helpful to understanding of the growth mechanism, we carried out a systematic study using a commercial mass spectrometer (Aeolos mass spectrometer, model MS 403C) in conjunction with an apparatus (model STA 449C/6/G Jupiter) consisting of a thermogravimeter (TG) and a differential scanning calorimeter (DSC). The whole process is monitored from the early temperature raising stage up to final heating stage. The specific aim is to understand the chemical reactions at different stages of the process. We recorded the change in the mass of the sample, the calorific change in the system and the chemical species existing in the experimental chamber. In such an experiment, a pure (99\%) Mo sheet, i.e., the evaporation source, was put in the $\mathrm{Al}_{2} \mathrm{O}_{3}$ crucible set on the balance housed in the vacuum chamber of the TG/DSC apparatus. After the vacuum system was evacuated to $\sim 1.2 \mathrm{~Pa}$, high purity $\mathrm{Ar}$ gas $(99 \%)$ was injected into the vacuum chamber with a flow rate of 200 sccm until it reached atmosphere pressure. Then, the chamber was evacuated again. When the chamber pressure reached $\sim 1.2$ $\mathrm{Pa}, \mathrm{Ar}$ gas with the same flow rate was injected again, just for $20 \mathrm{~s}$. Then, the sample was heated to $1873 \mathrm{~K}$ with a rate of 20 $\mathrm{K} / \mathrm{min}$. Meanwhile, the mass spectrometer connected with the TG/DSC apparatus through a capillary was used to monitor the chemical species in the chamber.

Field Emission Measurements. The field emission studies were carried out in a chamber having vacuum of $\sim 4.0 \times 10^{-5}$ $\mathrm{Pa}$ at room temperature. The sample was first adhered to the surface of an oxygen free high conductivity copper disk using silver paint. A manipulator (Huntington PM-600-T) was used to control the distance $(d)$ between the anode and the cathode. A vacuum gap of $0.20 \mathrm{~mm}$ was used when we measured the field emission properties of Mo nanowire bundles. A CCD was used to record the spatial distribution of emission sites.

\section{Results and Discussion}

Figure 1 shows typical SEM images of very high-density Mo nanowires grown on stainless steel foil with evaporation temperature of $\sim 1623 \mathrm{~K}$ and substrate temperature of $\sim 1523$ K. Figures 1a is a low magnification SEM image of the Mo nanowires. The nanowire arrays with length of $\sim 10 \mu \mathrm{m}$ grew uniformly on the substrate over a large area. Figure $1 \mathrm{~b}$ is a higher magnification SEM image from the rectangular-enclosed area of $(B)$ in Figure 1a, showing that the nanowires are almost vertically aligned. A higher magnification SEM image in Figure $1 \mathrm{c}$ is from the rectangular-enclosed area of $(C)$ in Figure 1a, showing that the nanowires in the top region are aligned and well separated from each other. The surface of the nanowires is rather rough and there are many little branches along the growth axis of the nanowires. The enlarged SEM image of the rectangular-enclosed area of $D$ in Figure 1a is shown in Figure $1 \mathrm{D}$, revealing that the nanowires in the bottom region are random. A typical XRD spectrum of the nanowires on the stainless steels is shown in Figure 2. In the spectra, the four peaks can be indexed to be (110), (200), (211), and (220) atomic planes of body centric cubic (bcc) Mo [space group: $\operatorname{Im} 3 \mathrm{~m}$ (229), with lattice constant $a=0.31472 \mathrm{~nm}$ (JCPDS card No. 42-1120)]. Meanwhile, the absence of other peaks demonstrates that the samples are dominated by pure Mo. Transmission electron microscopy (TEM) provides further insight into the morphology and microstructure of the as-synthesized Mo nanowires. Figure 3 a shows a low magnification TEM image of a Mo nanowire, revealing that the surface of the nanowire along the length is nonuniform, and there are some nanoparticles adhere to the nanowire. The selected area electron diffraction (SAED) pattern (inset in Figure 3a) of the nanowire indicates that the nanowire is polycrystalline but has a textured structure. Figure $3 \mathrm{~b}$ shows a high-resolution transmission electron microscopy (HRTEM) image of a Mo nanowire, and Figure $3 \mathrm{c}$ is an enlarged image from the rectangular-enclosed area in Figure $3 \mathrm{~b}$, showing that the Mo nanowire is crystalline, and the 


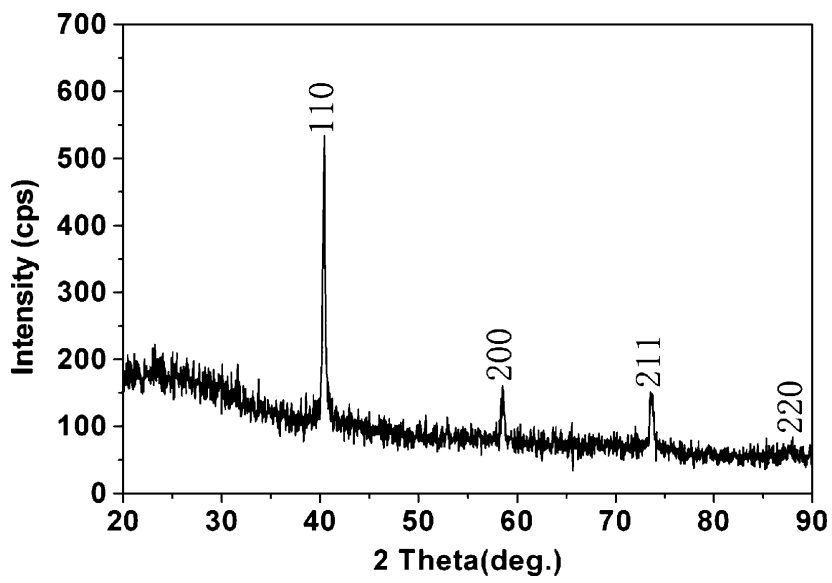

Figure 2. X-ray diffraction (XRD) pattern of the Mo nanowires.

interplanar spacing is $\sim 0.22 \mathrm{~nm}$, corresponding to the $\{110\}$ planes of bcc Mo.

In our study, we found that, with same evaporation source temperature, a decrease in substrate temperature can result in poor quality metal nanowire (Supporting Information). However higher growth temperature will enhance the crystalline of the Mo nanowires. Typically, when the source temperature and substrate temperature are both high enough, we can get single crystalline Mo nanowires. Figure 4 shows nanowire samples grown on single crystalline (0001) plane of $\alpha-\mathrm{Al}_{2} \mathrm{O}_{3}$ substrate with source temperature and substrate temperature of $\sim 1773$ and $\sim 1665 \mathrm{~K}$, respectively. The SEM image (Figure 4a) shows that the nanowires have diameters of $\sim 200 \mathrm{~nm}$. The XRD pattern in Figure $4 \mathrm{~b}$ indicates the Mo nanowires have singlephase bcc structure. The (0006) peak came from the single crystalline $\alpha-\mathrm{Al}_{2} \mathrm{O}_{3}$ substrate. The TEM images and corresponding SAED patterns (Figure $4 \mathrm{c}-\mathrm{e}$ ) reveal that the nanowires are of single crystalline with growth direction of $\langle 111\rangle$ and $\langle 211\rangle$. The $\langle 111\rangle$ growth direction is reasonable, because the epitaxial relationship between Mo and (0001) $\alpha-\mathrm{Al}_{2} \mathrm{O}_{3}$ substrate is [111] $\mathrm{Mo}||[0001]_{\mathrm{Al}_{2} \mathrm{O}_{3}}$, and $(211)_{\mathrm{Mo}}$ with $(10 \overline{1} 0)_{\mathrm{Al}_{2} \mathrm{O}_{3}}$ planes as well as $(110)_{\mathrm{Mo}}$ with $(\overline{2} 110)_{\mathrm{Al}_{2} \mathrm{O}_{3}}$ have the best match interplanar distance. ${ }^{11}$ However, the $\langle 211\rangle$-growth direction is not fully understood. We also grew single crystalline tungsten nanowires on the (0001) $\alpha-\mathrm{Al}_{2} \mathrm{O}_{3}$ substrates with $\langle 111\rangle$ and $\langle 211\rangle$ growth directions (support information 3 (HRTEM images of tungsten nanowires)). The enhancement of the crystallinity of Mo nanowires with increased growth temperature is reasonable, because high temperature will increase the transport ability and the mobility of the Mo atoms.

It should be noted that when hydrogen gas was introduced during the entire growth process, we could not grow Mo nanowires. That means under conditions of high temperature and high vacuum, although Mo vapor can possible evaporate from the Mo boat by physical evaporation, the Mo vapor cannot reach super saturation to form Mo nanowires. So we suspect that there are other ways to provide Mo source to grow Mo nanowires. To find out the possible ways, we first studied the chemical species available in the vapor in the growth chamber at different temperatures using the system of mass spectrometer with TG-DSC. Figure 5a shows the typical simultaneous TGDSC curves up to the source temperature of $1873 \mathrm{~K}$. After a short period of thermal balance before $400 \mathrm{~K}$, the DSC curve gives a very broad exothermic peak with several small fine exothermic peaks. All of the exothermic peaks correspond to the oxidation of Mo sample. The reactions may be as follows: $\mathrm{Mo}(\mathrm{s})+\mathrm{O}_{2} \rightarrow \mathrm{MoO}_{x}(\mathrm{~s})$. The $\mathrm{O}_{2}$ was residual in the chamber. The calculation of the free Gibbs energy $(\Delta G)$ by using the thermodynamic data ${ }^{12}$ (Supporting Information, Free Gibbs energy diagram) reveals that all kind of molybdenum oxides including $\mathrm{MoO}_{3}, \mathrm{Mo}_{9} \mathrm{O}_{26}, \mathrm{Mo}_{8} \mathrm{O}_{23}, \mathrm{Mo}_{4} \mathrm{O}_{11}$, and $\mathrm{MoO}_{2}$ could be formed during the oxidation process. We also used XRD to analyze of molybdenum oxides that were formed on the Mo sheet at different temperature (Supporting Information, series $\mathrm{XRD}$ results), the XRD results reveal that at low-temperature range $(\sim 873-973 \mathrm{~K}), \mathrm{MoO}_{3}$ were formed on the surface of Mo sheet, and, however, at high temperature range (>1310 K), $\mathrm{MoO}_{2}$ were formed on the surface of Mo sheet. Simultaneous scan of mass spectra of all the evolved species was done at $\sim 1773 \mathrm{~K}$, and the result is shown in Figure 5b. The single ion $\left(\mathrm{Mo}^{+}, \mathrm{m} / z\right.$ 94-98.5) and a number of fragments due to dissociation and ionization $\left(\mathrm{MoO}_{2}{ }^{+}, \mathrm{m} / \mathrm{z}, 128-134, \mathrm{MoO}_{3}{ }^{+}, \mathrm{m} / \mathrm{z}\right.$ 144-148) are clearly detected (see Supporting Information (table listing the isotopes and figure showing the baseline spectrum).

We now discuss the origin of $\mathrm{Mo}, \mathrm{MoO}_{2}$, and $\mathrm{MoO}_{3}$ species that were detected by the mass spectroscopy. First, Mo and $\mathrm{MoO}_{2}$ were evaporated from the Mo sample and its oxidized surface layer, respectively; these obviously are possible under vacuum conditions. But $\mathrm{MoO}_{3}$ vapor cannot come from evaporation of the sample, because its boiling point $(\sim 1428$ $\mathrm{K})$ is below the sample temperature so that it cannot exist in a solid state in the sample (see also Supporting Information, series of XRD results). Second, according to the systematic calculation data of Gibbs free energy (see table in Supporting Information), the following chemical reactions can happen spontaneously to give rise to $\mathrm{MoO}_{2}$ and $\mathrm{MoO}_{3}$ vapors:

$$
\begin{aligned}
& \mathrm{Mo}(\mathrm{s})+\mathrm{O}_{2}(\mathrm{v}) \rightarrow \mathrm{MoO}_{2}(\mathrm{v}), \quad \Delta G=-69 \mathrm{~kJ} /(\mathrm{mol} \mathrm{K}) \\
&(T=1623 \mathrm{~K})(1) \\
& 2 \mathrm{Mo}(\mathrm{s})+3 \mathrm{O}_{2}(\mathrm{v}) \rightarrow 2 \mathrm{MoO}_{3}(\mathrm{v}), \quad \Delta G=-264 \mathrm{~kJ} /(\mathrm{mol} \mathrm{K}) \\
&(T=1623 \mathrm{~K})(2)
\end{aligned}
$$

From the result in eq 1 and the mass spectrum results in Figure 5b, we can figure out that the $x$ in $\mathrm{MoO}_{x}$ is 2, which was proposed to be precursor of the growth of $\mathrm{MoO}_{2}$ nanowires in low temperature. ${ }^{6 f}$ We now move to explain how these vapors may contribute to the growth of Mo nanowires. Again, according to the systematic calculated data of Gibbs free energy (see Supporting Information), the growth of the Mo nanowires may be by deposition of Mo atoms coming from the decomposition of $\mathrm{MoO}_{2}$ adatoms during a condensation process through the following chemical reaction:

$$
\begin{aligned}
3 \mathrm{MoO}_{2}(\mathrm{v}) \rightarrow \mathrm{Mo}(\mathrm{s})+2 \mathrm{MoO}_{3}(\mathrm{v}), & \\
\Delta G & =-341 \mathrm{~kJ} /(\mathrm{mol} \mathrm{K}) \quad(T=1523 \mathrm{~K})
\end{aligned}
$$

The Gibbs free energy value is negative, so the chemical reaction can occur spontaneously. When hydrogen gas was introduced during the growth process we could not grow Mo nanowires, because the hydrogen suppressed the formation of $\mathrm{MoO}_{2}$ vapor, indicating the process in eq 3 is dominant for the growth of Mo nanowires. The decomposition reaction for the formation of Mo in this work is similar to the formation of $\mathrm{W}$ with little difference, where $\mathrm{WO}_{2}$ was decomposed to form $\mathrm{W}$ and $\mathrm{O}_{2}{ }^{8 \mathrm{c}}$

Here we come to explain why Mo nanowires are detected in our sample instead of Mo oxide nanowires. In our experiments, the substrates temperatures were at least $\sim 1453 \mathrm{~K}$, and the temperature was higher than the boiling point of $\mathrm{MoO}_{3}$ (boiling point: $1428 \mathrm{~K}$ ), thus there is no condensation of $\mathrm{MoO}_{3}$ vapor on the substrates. Furthermore, Figure 5c shows a plot of Gibbs free energy vs temperature for the condensation process of $\mathrm{MoO}_{2}$ 


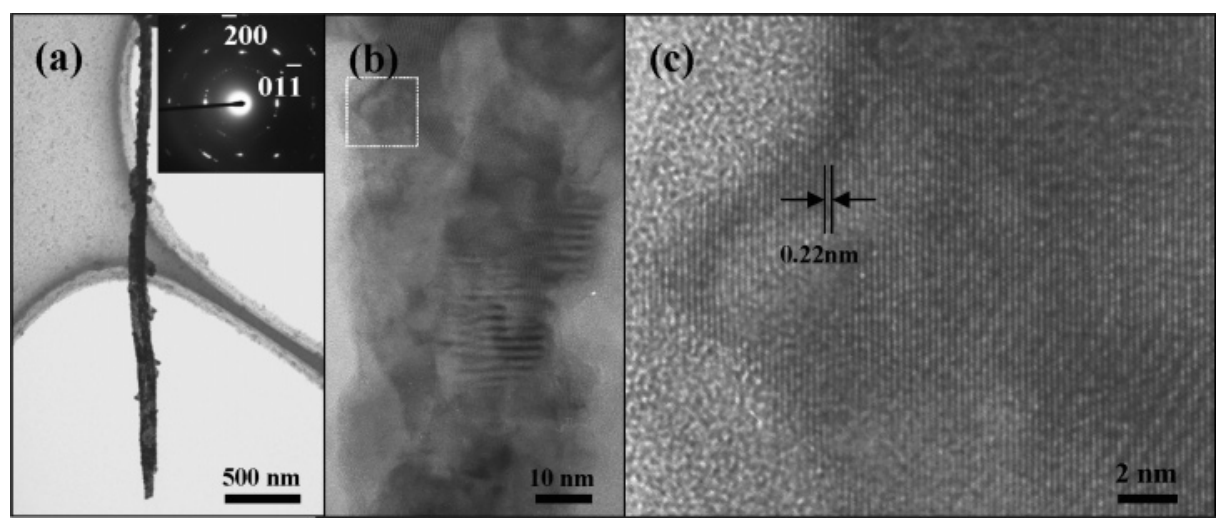

Figure 3. (a) Low magnification TEM image of a Mo nanowires, inset shows the corresponding SAED pattern of the nanowire; (b) Highresolution TEM image of a Mo nanowire. (c) Enlarged image of the rectangle-enclosed area in part $b$.

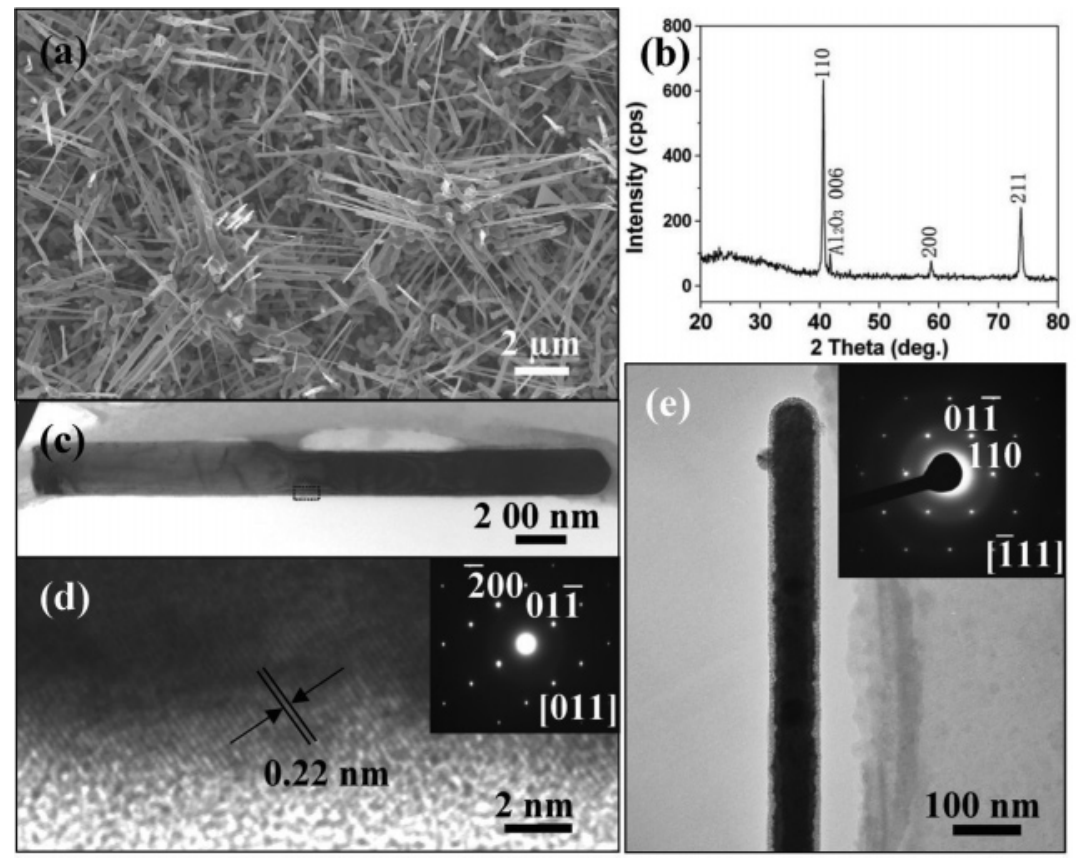

Figure 4. (a) SEM images of Mo nanowires grow on single crystal (0001) $\alpha-\mathrm{Al}_{2} \mathrm{O}_{3}$ substrate with source temperature and substrate temperature of $\sim 1773$ and $\sim 1665 \mathrm{~K}$, respectively. (b) XRD pattern of the sample. (c) TEM image of a Mo nanowire. (d) High-resolution TEM image of rectangle-enclosed area in part $\mathrm{c}$. The corresponding SAED in the inset indicates the nanowire is single crystalline with $\langle 111\rangle$ growth direction. (e) TEM image and corresponding SAED pattern of anther single crystalline Mo nanowire with $\langle 211\rangle$-growth direction.

vapor. Since the Gibbs free energy values are negative, $\mathrm{MoO}_{2}$ vapor can condense on our substrates. However, Figure 5c also reveals that, as the temperature increases, the absolute value of the Gibbs free energy decreases, meaning that when the temperature goes higher, the condensation process of $\mathrm{MoO}_{2}$ will be difficult. In our experimental case, as the substrate temperature is high enough, if $\mathrm{MoO}_{2} / \mathrm{Mo}$ ratio is very small, it is difficult to detect the $\mathrm{MoO}_{2}$ signal by XRD, because the peaks from $\mathrm{MoO}_{2}$ may be merged into the noise. Finally, the introduction of the $\mathrm{H}_{2}$ gas in the cooling process also can improve the purity of the products (Supporting Information, XRD pattern of a Mo nanowires sample which was synthesized without introduction of the $\mathrm{H}_{2}$ gas in the cooling process).

Having described the chemistry-governed growth of Mo nanowires, here we discuss how the aligned nanowires were formed from the point of view of physics. A piece of Mo nanowire bundles lifted by tweezers is given in Figure 6a. A high magnification SEM image from the rectangular-enclosed area in Figure 6a is presented in Figure 6b, revealing a layer of nanoparticles between the top treeliked nanowire bundle layer and the underneath substrate. On the basis of the above observation, we can interpret how the nanowire bundles were formed. A competition growth process can explain the growth of the nanowires. ${ }^{13,14}$ Initially, a layer of nanoparticles was first formed on the surface of the substrate (Figure 6d). Next, treelike Mo nanowire bundles start to grow (Figure 6e). The nanowires of the treelike nanowire bundles have no orientation ordering. As a result, the growth of the nanowires follows two major steps: First, the nanowires grow parallel or nearly parallel to the substrate surface will terminate due to limited space when they hit other nanowires (Figure 1d). Second, the nanowires growing along the normal or nearly normal direction of the substrate continue to grow to form long-length nanowires (Figure 1c). Thus, the nanowires tend to be aligned as the growth continues (Figure 6f).

Figure 7a shows a typical plot of emission current density, $J$, vs applied field curve obtained at a vacuum gap of $0.2 \mathrm{~mm}$. A turn-on filed of $5.0 \mathrm{MV} / \mathrm{m}$ and a threshold field of 9.9 MV/m were obtained, these values are much lowers then that of the classical Spindt Mo tips. ${ }^{15}$ The Fowler-Nordheim $(\mathrm{F}-\mathrm{N})$ plot [top-left inset in Figure 7a] exhibits linear dependence, indicating that the emission is consistent with the FN 

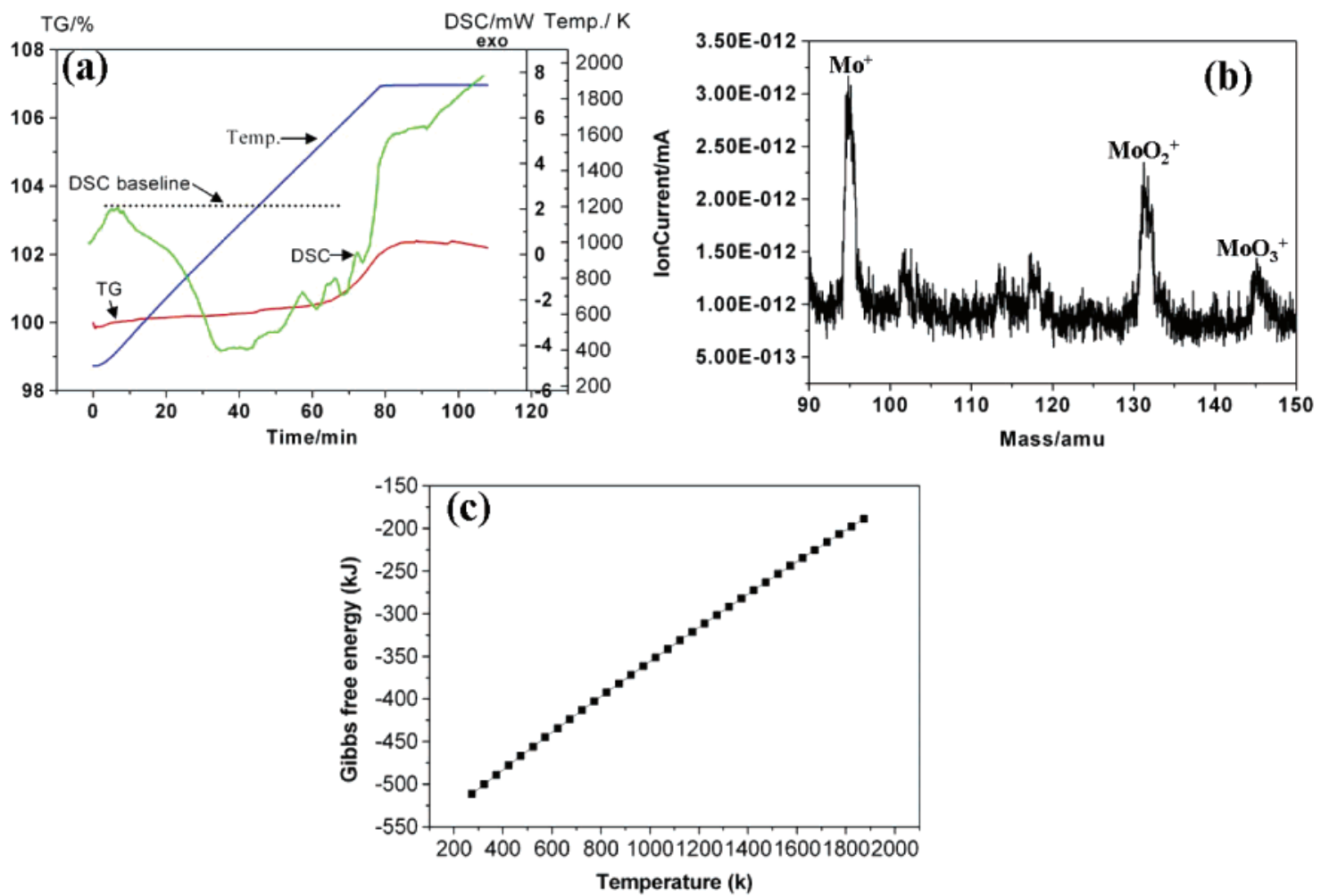

Figure 5. (a) Simultaneous TG-DSC measured curves. (b) Typical mass spectrum recorded at 1773 K. (c) Gibbs free energy vs temperature plot.
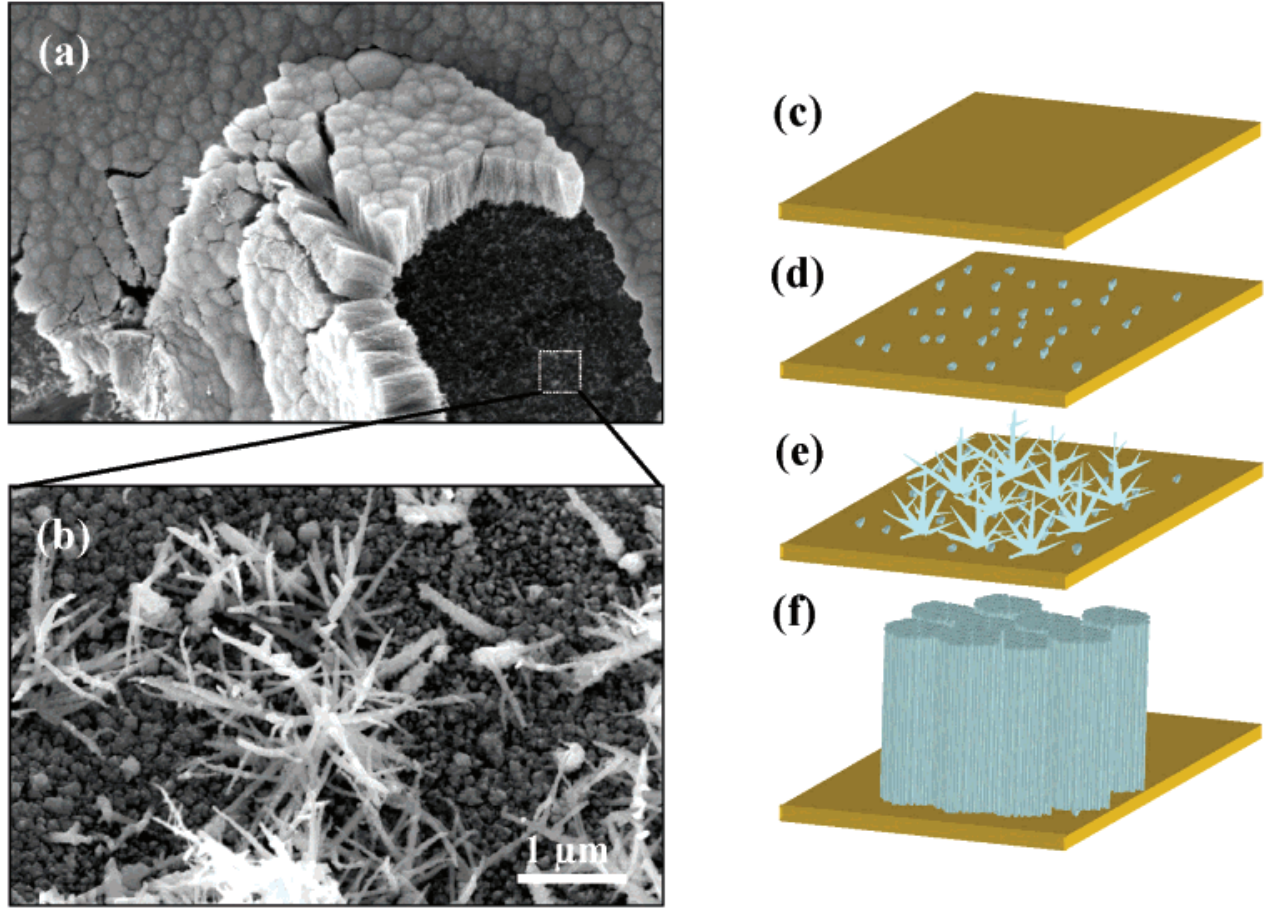

Figure 6. (a) Low magnification SEM image of bunches of Mo nanowire bundles that were lifted by a tweezer. (b) Enlarged SEM images of the rectangle-enclosed area in part a. $(b-e)$ Schematic diagram of the growth process of the Mo nanowire bundles.

mechanism. The bottom-right inset in Figure 7a is the CCD images of the sample and the spatial distribution of the emission sites $\left(11.2 \mathrm{MV} / \mathrm{m}\right.$ and $\left.20 \mathrm{~mA} / \mathrm{cm}^{2}\right)$. A comparison between the CCD camera images of the sample and the emission site distribution images shows that the distribution in emission sites is uniform. The above findings show the potential application of the nanowires as cold cathode electron emitters. We also grow Mo nanowires on the $2.5 \mathrm{~cm}$ long stainless steel wires as electron source for luminescent tube (support information 9
(Sketch of the structure of the luminescent tube)). Figure $7 \mathrm{~b}$ shows a typical emission current, $I$, vs applied field curve of the Mo nanowire luminescent tube. The insets in Figure $7 \mathrm{~b}$ are a digital camera photograph of the luminescent tube and the corresponding CCD image of the working luminescent tube, respectively. The intensity of the whole tube screen is quite homogeneous. The brightness of such a tube can be as high as $\sim 30000 \mathrm{~L} / \mathrm{m}^{2}$, which reaches the basic requirement for practical application. 

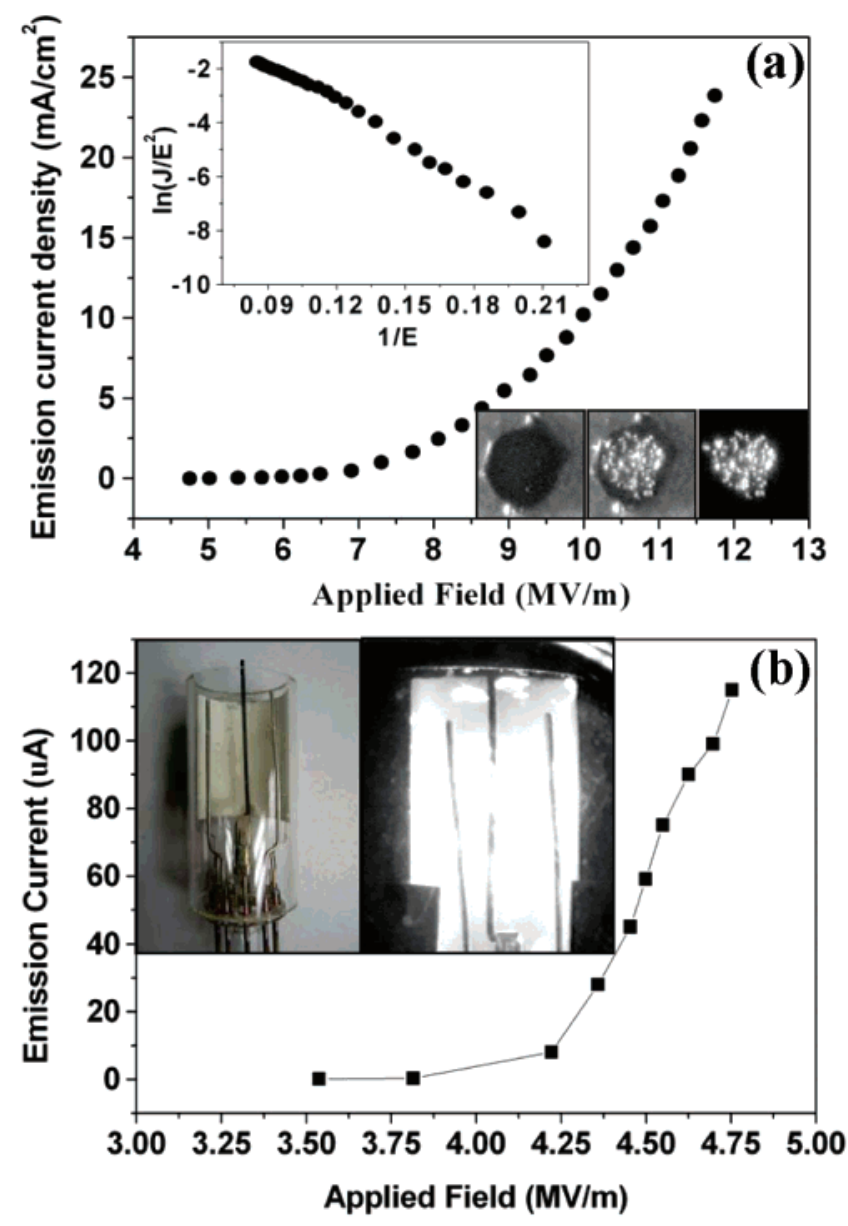

Figure 7. (a) Field emission current density vs electric field $(J-E)$ plot of Mo nanowires. Left-hand inset: corresponding $F-N$ plot. Righthand inset: CCD images of the sample and the spatial distribution of the emission sites $\left(11.2 \mathrm{MV} / \mathrm{m}\right.$ and $\left.20 \mathrm{~mA} / \mathrm{cm}^{2}\right)$ when the light is on and off. (b) $I-E$ curve of the Mo nanowire luminescent tube, where the inset shows a digital camera photograph of the luminescent tube and a CCD image of the working state of the luminescent tube.

\section{Conclusion}

Large-area aligned Mo nanowires have been grown on stainless steel substrates by a high temperature chemical vapor deposition approach. This is a simple and scalable new approach for synthesis of aligned metal nanowires. At relatively low growth temperatures, the nanowires are polycrystalline; an increase in growth temperature enhances the crystallinity of the nanowires. The detailed physical and chemical growth processes regarding the formation of the nanowires have been investigated using mass spectroscopy, thermogravimetry and differential scanning calorimetry analysis, as well as structure analysis by electron microscopy. By combining the information provided by all of these techniques, we found that the growth relies on the decomposition of $\mathrm{MoO}_{2}$ adatoms/vapor through condensation of its vapor at high substrate temperatures. The aligned growth is a result of competing growth with the nanowires normal to the substrate surface reaching the final growth front. The field emission measurement and the vacuum luminescent tube study show that the Mo nanowires have potential application as electron emitters.

Acknowledgment. N.X., S.D., and J.C. gratefully acknowledge the financial support of the project from the National Natural Science Foundation of China (Grant Nos. 50021202, 50329201, 90201020, and 60571035), Science and Technology
Ministry of China (Grant Nos. 2003CB314700, 2002AA313010, and 2001CCA04400), Education Ministry of China, Science and Technology Department of Guangdong Province, Education Department of Guangdong Province, and Science and Technology Department of Guangzhou City. Z.L.W. expresses thanks for the support from NASA Vehicle Systems, Department of Defense Research and Engineering (DDR\&E), and the Defense Advanced Research Projects Agency (Award No. N66001-041-8903). J.Z. expresses thanks for the KAISI FUND from SunYat-Sen (Zhongshan) University and Rusen Yang's help.

Supporting Information Available: Figures showing the schematic diagram of the synthesis apparatus, SEM and TEM images of Mo nanowire bundles, SEM images of Mo nanowires, TEM and HRTEM images of the Mo nanowires, and TRTEM images and corresponding FFT of single crystalline W nanowires, tables of Gibbs free energy vs temperature, figures of XRD analysis of molybdenum oxides, a table of molybdenum isotopes and possible mass numbers for oxides, and figures showing the base mass spectrum, XRD pattern of a Mo nanowires sample, and a sketch of the structure of the luminescent tube. This material is available free of charge via the Internet at http:// pubs.acs.org.

\section{References and Notes}

(1) Patolsky, F.; Weizmann, Y.; Willner, I. Nat. Mater. 2004, 3, 692.

(2) (a) Ye, X. R.; Lin, Y. H.; Wang, C. M.; Wai, C. M. Adv. Mater 2003, 15, 316. (b) Martin, C. R. Science 1994, 266, 1961. (c) Brumlik, C, J.; Menon, V. P.; Martin, C. R. J. Mater. Res. 1994, 9, 1174. (d) Keating, C. D.; Natan, M. J. Adv. Mater. 2003, 15, 451. (e) Vila, L.; Vincent, P.; Pra, L. D. D.; Pirio, G.; Minoux, E.; Gangloff, L.; Demoustier-Champagne, S.; Sarazin, N.; Ferain, E.; Legras, R.; Piraux. L.; Legagneux, P. Nano. Lett. 2004, 4, 521. (f) Teinhart, M. S.; Jia, Z. H.; Schaper, A. K.; Wehrspohn, R. B.; G.osele, U.; Wendorf, J. H. Adv. Mater. 2003, 15, 706. (g) Fahmi, A. W.; Braun, H. G.; Stamm, M. Adv. Mater. 2003, 15, 1201. (h) Wirtz, M.; Martin, C. R. Adv. Mater. 2003, 15, 455. (i) Braun, E.; Eichen, Y.; Sivan, U.; Yoseph, G. B. Nature (London) 1998, 391, 775. (j) Chen, J.; Tao, Z. L.; Li, S. L. J. Am. Chem. Soc. 2004, 126, 3060. (k) Zhang, M. Z.; Lenhert, S.; Wang, M.; Chi, L. F.; Lu, N.; Fuchs, H.; Ming, N. B. Adv. Mater. 2004, 16, 409 .

(3) (a) Choi, H.; Park, S. H. J. Am. Chem. Soc. 2004, 126, 6248. (b) Vivekchand, S. R. C.; Gundiah, G.; Govindaraj, A.; Rao, C. N. R. Adv. Mater., 2004, 16, 1842.

(4) (a) Sun, Y. G.; Mayers, B.; Xia, Y. N. Adv. Mater. 2003, 15, 641. (b) Sun, Y. G.; Mayers, B.; Xia, Y. N. Nano Lett. 2002, 2, 481.

(5) (a) Sun, Y. G.; Gates, B.; Mayers, B.; Xia, Y. N. Nano. Lett. 2002, 2, 165. (b) Sun, Y. G.; Xia, Y. N. Adv. Mater. 2002, 14, 833. (c) Busbee, B. D.; Obare, S. O.; Murphy, C. J. Adv. Mater. 2003, 15, 414. (d) Xiong, Y. J.; Xie, Y.; Wu, C. Z.; Yang, J.; Li, Z. Q.; Xu, F. Adv. Mater. 2003, 15, 405. (e) Yen, M. Y.; Chiu, C. W.; Hsia, C. H.; Chen, F. R.; Kai, J. J.; Lee, C. Y.; Chiu, H. T. Adv. Mater. 2003, 15, 235.

(6) (a) Li, D.; Herricks, T.; Xia, Y. N, Appl. Phys. Lett. 2003, 83, 4586. (b) Li, Y. D.; Li, X. L.; Deng, Z. X.; Zhou, B. C.; Fan, S. S.; Wang, J. W.; Sun, X. M. Angew, Chem. Int. Ed. 2002, 41, 333. (c) Wang, J. W.; Li, Y. D. Adv. Mater. 2003, 15, 445. (d) Zach, M. P.; Ng, K. H.; Panner, R. M. Science 2000, 290, 2120. (e) Zach, M. P.; Inazu, K.; Ng, K. H.; Hemminger, J. C.; Penner, R. M. Chem. Mater. 2002, 14, 3206. (f) Zhou, J.; Xu, N. S.; Deng, S. Z.; Chen, J.; She, J. C.; Wang, Z. L. Adv. Mater. 2003, 15, 1835. (g) Chen, J.; Tao, Z. L.; Li, S. L. J. Am. Chem. Soc. 2004, 126, 3060.

(7) (a) Thong, J. T. L.; Oon, C. H.; Yeadon, M.; Zhang, W. D. Appl. Phys. Lett. 2002, 81, 4823. (b) Karabacak, T.; Wang, G. C.; Lu, T. M. J. Appl. Phys. 2003, 94, 7723. (c) Vivekchand, S. R. C.; Gundiah, G.; Govindaraj, A.; Rao, C. N. R. Adv. Mater. 2004, 16, 1842. (d) Song, M.; Mitsuishi, K.; Tanaka, M.; Takeguchi, M.; Shimojo, M.; Furuya, K. Appl. Phys. A: Mater. Sci. Process. 2005, 80, 1431.

(8) (a) Lee, Y. H.; Choi, C. H.; Jang, Y. T.; Kim, E. K.; Ju, B. K.; Min, N. K.; Ahn, J. H. Appl. Phys. Lett.. 2002, 81, 745. (b) Liu, Z. W.; Bando, Y. Adv. Mater. 2003, 15, 303. (c) Vaddiraju, S.; Chandrasekaran, H.; Sunkara, M. K. J. Am. Chem. Soc. 2003, 125, 10792. (d) Kong, X. Y.; Ding, Y.; Wang, Z. L. J. Phys. Chem. B 2004, 108, 570. (e) Wen, X. G.; Fang, Y. P.; Yang, S. H. Angew. Chem., Int. Ed. 2005, 44, 3562.

(9) Karabacak, T.; Mallikarjunan, A.; Singh, J. P.; Ye, D. X.; Wang, G. C.; Liu, T. M. Appl. Phys. Lett. 2003, 83, 3096.

(10) Spindt, C. A.; Brodiel, I.; Humphrey, L.; Westerberg, E. R. J. Appl. Phys. 1976, 47, 5248 . 
(11) Cillet, M.; Masek, K.; Lemire, C. Thin Solid Film 2003, 444,9

(12) (a) NASA Thermo Build, http://cea.grc.nasa.gov. (b) Chase, M W., Jr.; Davies, C. A.; Downey, J. R., Jr.; Frurip, D. J.; Mcdonald, R. A.; Syverud, A. N. JANAF Thermochemical Tables, 3rd ed.; J. Phys. Chem. Ref. Data 1985, 14, Suppl. 1.
(13) Wang, Z. L.; Bentley, J.; Clausing, R. E.; Heatherly, L.; Horton, L. L. J. Mater. Res. 1994, 9, 1552 .

(14) Li, D. C.; Dai, L. M.; Huang, S. M.; Mau, A. W. H.; Wang, Z. L. Chem. Phys. Lett., 2000, 316, 349.

(15) Spindt, C. A.; Brodie, I.; Humphrey, L.; Westerberg, E. R. J. Appl. Phys. 1976, 47, 5248. 